\title{
Evans Syndrome Secondary to Common Variable Immune Deficiency
}

\author{
James W. Antoon, MD, PhD, ${ }^{*}$ Diana Metropulos, $M D, \dagger$ and \\ Benny L. Joyner, Jr, MD, MPH
}

\begin{abstract}
Summary: Evans syndrome is an underdiagnosed condition consisting of simultaneous or sequential combination of autoimmune hemolytic anemia and immune-mediated thrombocytopenia. We report a case of severe Evans syndrome presenting as altered mental status, a rare presenting sign of the disease. This case highlights the difficulty in diagnosing Evans syndrome and provides a review of the literature and management strategies for treating the disorder.
\end{abstract}

Key Words: Evans syndrome, anemia, immunodeficiency, altered mental status, pediatrics

( J Pediatr Hematol Oncol 2016;38:243-245)

\section{OBSERVATIONS}

A 13-year-old white boy with a history of idiopathic thrombocytopenic purpura was transferred to our facility following an admission at an outside hospital for altered mental status (AMS). His history of present illness was significant for 3 days of headache, photosensitivity, decreased appetite, and fatigue as well as 2 days of jaundice, pale skin color, and increased bruising in his upper extremities. On day of admission he was found to be drowsy and unresponsive to simple commands. The patient had a history of idiopathic thrombocytopenic purpura, described as intermittent and self-limited petechiae for the previous 3 years. At the outside emergency room, the patient was intubated due to his AMS. Bloodwork was performed and he received intravenous fluids and empiric antibiotics. The patient was then transferred to the pediatric intensive care unit at our institution.

The patient was febrile, tachycardic, and hypotensive on admission. Upon physical examination, the patient was orally intubated and sedated. He withdrew from painful stimuli but remainder of neurological examination was limited due to sedatives. Pupils were equal round and reactive to light with icteric conjunctivae. No palpable lymphadenopathy was present. Respiratory examination demonstrated ventilated breath sounds that were mildly diminished at the bases. Cardiovascular examination was significant for tachycardia and a regular rhythm without murmurs, rubs, or gallops. Hypoactive bowel sounds were

Received for publication February 12, 2015; accepted January 28, 2016. From the *Department of Pediatrics, Children's Hospital, University of Illinois Hospital \& Health Sciences System; †Department of Pediatrics, Rush University Medical Center, Chicago, IL; and $\ddagger$ Departments of Pediatrics, University of North Carolina School of Medicine, Chapel Hill, NC

The authors declare no conflict of interest.

Reprints: James Antoon, MD, PhD, Department of Pediatrics, Children's Hospital, University of Illinois Hospital \& Health Sciences System, 840 S. Wood Street, Chicago, IL 60612 (e-mail: jantoon@uic.edu). noted with hepatomegaly and splenomegaly present. Skin was pale and jaundiced throughout with purpura noted over lower extremities, abdomen, and arms and petechiae over the anterior thighs bilaterally.

Initial laboratory values were significant for hypoalbuminemia, hypoproteinemia, hyperbilirubinemia, mild transaminitis, elevated lactate dehydrogenase and uric acid, and elevated inflammatory markers. Hematological studies were notable for white blood cells $32.7 / \mathrm{mm}^{3}$ (51\% neutrophils, $16 \%$ bands, $11 \%$ lymps), hemoglobin $3.7 \mathrm{~g} / \mathrm{dL}$, hematocrit $10.5 \mathrm{~g} / \mathrm{dL}$, platelets 481 , red cell distribution width $20.1 / \mathrm{mm}^{3}$, mean corpuscular volume 66.5 , with marked polychromasia and spherocytes. Activated partial thromboplastin time 29.9 seconds, international normalized ratio 1.7, and prothrombin time 18.4 seconds. Direct coombs, anti-human globulin, anti-IgG, and anticompliment were all positive. Head computed tomography (CT) and magnetic resonance imaging revealed a likely calcification or microhemorrhage in the left caudate and posterior temporal lobe. Abdominal CT and abdominal ultrasound were consistent with hepatosplenomegaly and peripancreatic lymphadenopathy (Fig. 1). Chest radiograph was with normal limits. Electroencephalography did not show seizure activity.

Initial management at our institution consisted of packed red blood cell transfusion, high-dose methylprednisolone, intravenous immunoglobulin (IVIG), prophylactic famotidine, and empiric treatment with vancomycin, ceftriaxone, and acyclovir. Pediatric infectious disease, hematology, immunology, and rheumatology were

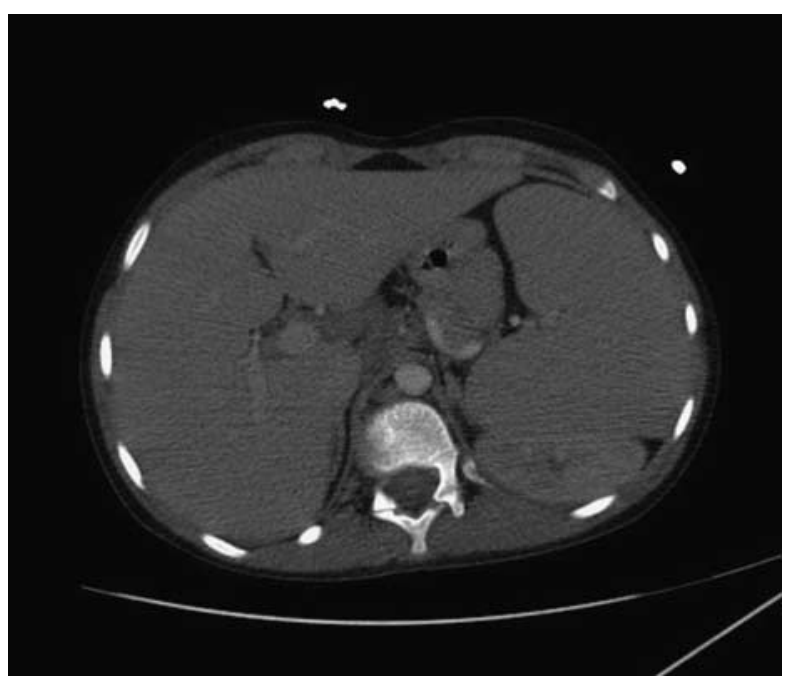

FIGURE 1. Computed tomography of the abdomen demonstrating hepatosplenomegaly. 
consulted. On hospital day 1 the patient's mental status gradually improved and he was extubated. Antimicrobials were discontinued when cultures and CSF studies returned negative on hospital day 2 . The patient's mental status returned to baseline on hospital day 2 following improvement of his anemia.

Given the patient's autoimmune hemolytic anemia and history of immune-mediated thrombocytopenia, a diagnosis of Evans syndrome was made. The patient's AMS was believed to be due to his severe anemia rather than the possible microhemorrhage found on CT. Following his clinical improvement, steroids were discontinued and he was started on mycophenolate mofetil (MMF). An extensive workup was performed while in the hospital to determine the underlying cause of his Evans syndrome. The patient was eventually discharged home 11 days after admission on a maintenance regimen of MMF. Several weeks after discharge, immunologic studies drawn before immune therapties returned revealing underlying diagnosis of common variable immune deficiency. The diagnosis was made due to diminished circulating B cells, decreased serum IgG and IgA, and subtherapeutic response to immunizations.

\section{CONCLUSIONS}

The case above highlights the difficulty in diagnosing Evans syndrome. The condition was first described in 1949 by Evans and Duane, who reported on 24 patients with acquired hemolytic anemia and thrombocytopenic purpura. ${ }^{1}$ Evans syndrome is currently defined as the simultaneous or sequential combination of autoimmune hemolytic anemia and immune mediated thrombocytopenia with a positive direct antiglobulin and exclusion of other independent causes of the patient's symptoms. Due to the rarity of the condition, epidemiological data is lacking on the condition and the prevalence and incidence of Evans Syndrome. Interestingly, a retrospective review of 237 cases of pediatric autoimmune hemolytic anemia revealed a diagnosis of Evans syndrome in $37 \%$ of cases. ${ }^{2}$ The disorder is likely underdiagnosed and has been described in all sexes, ages, and ethnic groups.

Evans syndrome is believed to be due to either primary or secondary dysregulation of both the cellular and humoral immune responses. Approximately $50 \%$ of cases are primary or idiopathic in origin. However, Evans syndrome is believed to be a phenotypic bicytopenic presentation of an underlying immune disease process and primary or idiopathic cases are likely related to undiagnosed immune disorders. The secondary causes of Evans syndrome are broad and often involve immune-mediated pathologic disease processes (Table 1). The differential diagnosis in the workup of Evans syndrome includes ADAMS-13 deficiency, hemolytic uremic syndrome, Kasaback-Merritt syndrome, paroxysmal nocturnal hemoglobinuria, and primary autoimmune lymphoproliferative disorder. Retrospective review of Evans syndrome is complicated by evidence that previous cases of the condition may have been due to previously undiagnosed autoimmune lymphoproliferative disorder. ${ }^{3}$

Common variable immunodeficiency (CVID) can be difficult to diagnose, particularly in the setting of therapeutic immune suppression. The diagnosis of CVID is based on 4 criteria: age above 4 years, low serum $\operatorname{IgG}$ with or without low serum IgA, diminished antibody response to immunizations, and exclusion of other potential immune
TABLE 1. Secondary Causes of Evans Syndrome $e^{3-13}$

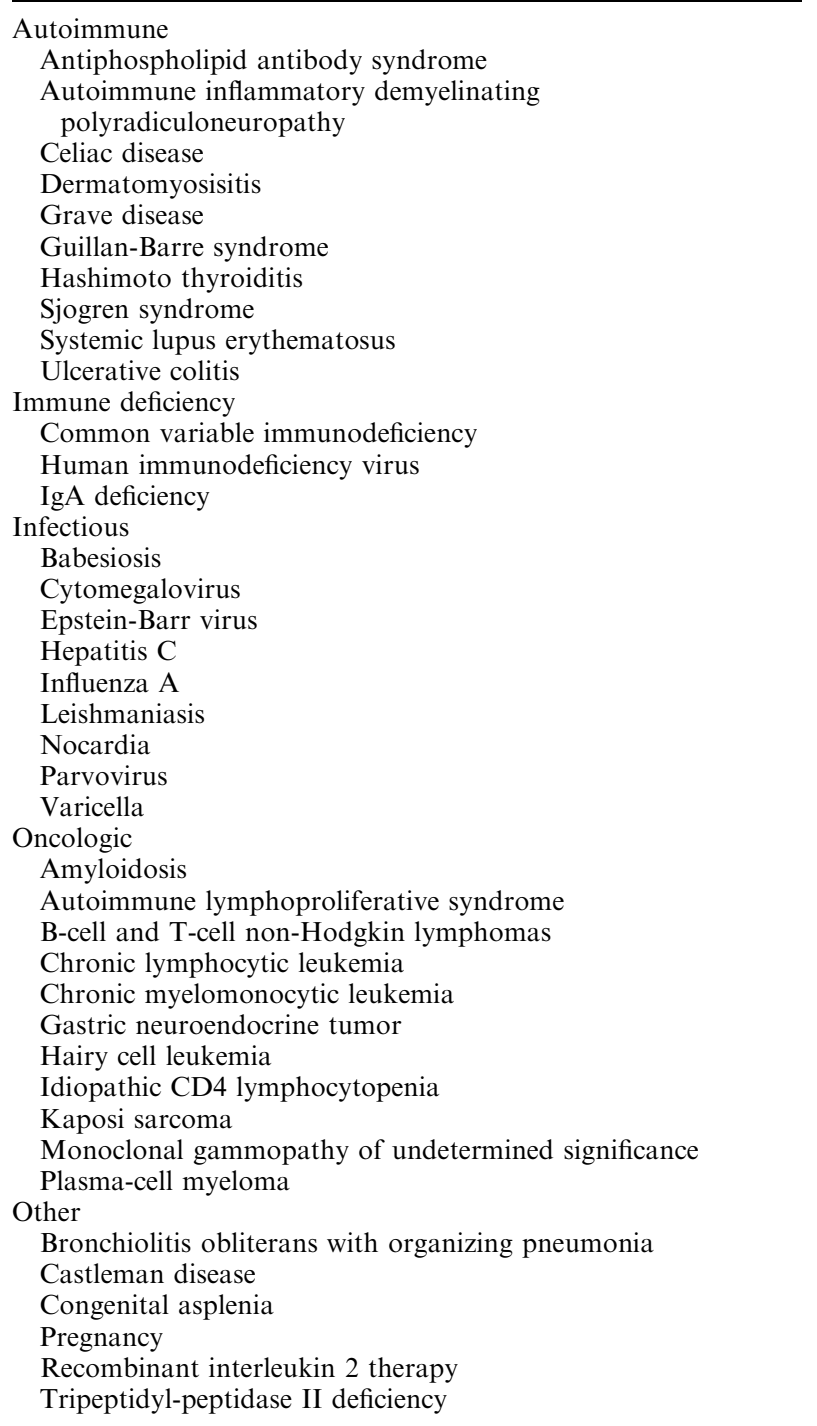

diseases. ${ }^{14}$ Therapeutic immune modulation for treatment of cytopenias or hemolysis can alter the immune systems assays. Patients will often receive IVIG, which will affect antibody levels, as all as other immunosuppressives that can affect humoral immune testing (steroids, MMF, rituximab, etc.). Diagnostic testing should be performed before immune suppression or specific therapies taken into account when treatment must be performed before workup.

Evans syndrome is characterized by frequent exacerbations that can spontaneously resolve and often follows a chronic waxing and waning course..$^{4,15,16}$ Diagnosis is often delayed, likely due to the rarity and variability in time course of the disease. The second presentation of cytopenia can occur months to years following the presentation of the first cytopenia, as was the case with our patient. Given the lack of controlled trials for Evans syndrome, therapeutic options remain highly variable (Table 2). ${ }^{5,16}$ The mainstay of treatment of Evans syndrome is immune suppression and most patients respond well to the treatment. First-line therapies consist of glucocorticoids and IVIG. Second-line 
TABLE 2. Treatment of Evans Syndrome ${ }^{4-6,12,17-23}$

First-line therapy
Glucocorticoids
Intravenous immunoglobulin
Second-line therapy
Alemtuzumab
Azathioprine
Cyclophosphamide
Cyclosporine
Danazol
Mycophenolate mofetil
Plasmapheresis
Rituximab
Tacrolimus
Vincristine
Refractory disease
Splenectomy
Hematopoietic bone marrow transplantation

therapy consists of immunosuppressive agents or chemotherapy. Severe or refractory disease can be treated with splenectomy or bone marrow transplantation.

Of note, patients with Evans syndrome secondary to CVID may have a varying response to treatment, including increased response to rituximab, compared with those with idiopathic disease. ${ }^{1,6,16,17,24,25}$ Splenectomy is controversial in CVID patients, who already have an underlying humoral disorder. Removal of the spleen, though an effective treatment for CVID-related cytopenias, comes at the risk of increased infection. One study of 48 patients with CVID and refractory cytopenia demonstrated splenectomy resulted in increased severe infections (meningitis, sepsis, fungal infections, peritonitis) and nonfatal surgical complications. ${ }^{26}$ CVID patients may also have systemic disease manifestations including gastrointestinal (both infectious and noninfectious), pulmonary symptoms (including both restrictive and obstructive lung disease), and other symptoms related to various infections. As noted above, CVID patients are at increased risk for infection, and it is possible that the immune response triggering hemolysis is due to recent or concurrent infectious processes. ${ }^{25,27,28}$ Therefore, CVID patient may require differential treatment and follow-up compared with those with traditional or idiopathic Evans syndrome.

\section{REFERENCES}

1. Michel M, Chanet V, Galicier L, et al. Autoimmune thrombocytopenic purpura and common variable immunodeficiency: analysis of 21 cases and review of the literature. Medicine. 2004;83:254-263.

2. Aladjidi N, Leverger G, Leblanc T, et al. New insights into childhood autoimmune hemolytic anemia: a French national observational study of 265 children. Haematologica. 2011;96:655-663.

3. Teachey DT, Manno CS, Axsom KM, et al. Unmasking Evans syndrome: T-cell phenotype and apoptotic response reveal autoimmune lymphoproliferative syndrome (ALPS). Blood. 2005;105:2443-2448.

4. Mathew P, Chen G, Wang W. Evans syndrome: results of a national survey. J Pediatr Hematol Oncol. 1997;19:433-437.

5. Wright DE, Rosovsky RP, Platt MY. Case records of the Massachusetts General Hospital. Case 36-2013. A 38-year-old woman with anemia and thrombocytopenia. $N$ Engl J Med. 2013;369:2032-2043.

6. Michel M, Chanet V, Dechartres A, et al. The spectrum of Evans syndrome in adults: new insight into the disease based on the analysis of 68 cases. Blood. 2009;114:3167-3172.
7. Dhingra KK, Jain D, Mandal S, et al. Evans syndrome: a study of six cases with review of literature. Hematology. 2008; $13: 356-360$.

8. Shatzel JJ, Donohoe K, Chu NQ, et al. Profound autoimmune hemolysis and Evans syndrome in two asplenic patients with babesiosis. Transfusion. 2015;55:661-665.

9. Stepensky P, Rensing-Ehl A, Gather R, et al. Early-onset Evans syndrome, immunodeficiency and premature immunosenescence associated with tripeptidyl-peptidase II deficiency. Blood. 2015;125:753-761.

10. Ebara S, Kagosima M, Marumo M, et al. Hairy cell leukemia accompanied by Evans syndrome. Rinsho ketsueki. 2014;55: 466-468.

11. Chung-Delgado K, Revilla-Montag A, Guillen-Bravo S, et al Refractory thrombocytopenia responds to octreotide treatment in a case of Evans syndrome with gastric neuroendocrine tumor. Case Rep Hematol. 2013;2013:391086.

12. Oh HJ, Yun MJ, Lee ST, et al. Evans syndrome following long-standing Hashimoto's thyroiditis and successful treatment with rituximab. Korean J Hematol. 2011;46:279-282.

13. Chen H, Jia XL, Gao HM, et al. Comorbid presentation of severe novel influenza A (H1N1) and Evans syndrome: a case report. Chin Med J. 2011;124:1743-1746.

14. Chapel H, Cunningham-Rundles C. Update in understanding common variable immunodeficiency disorders (CVIDs) and the management of patients with these conditions. $\mathrm{Br} J$ Haematol. 2009;145:709-727.

15. Wang WC. Evans syndrome in childhood: pathophysiology, clinical course, and treatment. Am J Pediatr Hematol Oncol. 1988;10:330-338

16. Seidel MG. Autoimmune and other cytopenias in primary immunodeficiencies: pathomechanisms, novel differential diagnoses, and treatment. Blood. 2014;124:2337-2344.

17. Mantadakis E, Danilatou V, Stiakaki E, et al. Rituximab for refractory Evans syndrome and other immune-mediated hematologic diseases. Am J Hematol. 2004;77:303-310.

18. Oda $\mathrm{H}$, Honda A, Sugita K, et al. High-dose intravenous intact $\mathrm{IgG}$ infusion in refractory autoimmune hemolytic anemia (Evans syndrome). J Pediatr. 1985;107:744-746.

19. Shanafelt TD, Madueme HL, Wolf RC, et al. Rituximab for immune cytopenia in adults: idiopathic thrombocytopenic purpura, autoimmune hemolytic anemia, and Evans syndrome. Mayo Clin Proc. 2003;78:1340-1346.

20. Chang DK, Yoo DH, Kim TH, et al. Induction of remission with intravenous immunoglobulin and cyclophosphamide in steroid-resistant Evans' syndrome associated with dermatomyositis. Clin Rheumatol. 2001;20:63-66.

21. Oyama Y, Papadopoulos EB, Miranda M, et al. Allogeneic stem cell transplantation for Evans syndrome. Bone Marrow Transplant. 2001;28:903-905.

22. Tabchi S, Hanna C, Kourie HR, et al. Successful treatment of Evans syndrome with tacrolimus. Invest New Drugs. 2015; 33:254-256

23. Farruggia P, Macaluso A, Tropia S, et al. Effectiveness of cyclosporine and mycophenolate mofetil in a child with refractory Evans syndrome. Pediatr Rep. 2011;3:e15.

24. Rao A, Kelly M, Musselman M, et al. Safety, efficacy, and immune reconstitution after rituximab therapy in pediatric patients with chronic or refractory hematologic autoimmune cytopenias. Pediatr Blood Cancer. 2008;50:822-825.

25. Cunningham-Rundles C, Bodian C. Common variable immunodeficiency: clinical and immunological features of 248 patients. Clin Immunol. 1999;92:34-48.

26. Wong GK, Goldacker S, Winterhalter C, et al. Outcomes of splenectomy in patients with common variable immunodeficiency (CVID): a survey of 45 patients. Clin Exp Immunol. 2013;172:63-72.

27. Quinti I, Soresina A, Spadaro G, et al. Long-term follow-up and outcome of a large cohort of patients with common variable immunodeficiency. J Clin Immunol. 2007;27:308-316.

28. Zullo A, Romiti A, Rinaldi V, et al. Gastric pathology in patients with common variable immunodeficiency. Gut. 1999;45:77-81. 